- Trabalho financiado com recursos da "Wenner-Gren Foundation for Anthropological Research", "Sigma-Xi Society for Scientific Research" $e$ Universidade de Indiana (EUA), através do "David C. Skomp Fund" do Departamento de Antropologia.

"Núcleo de Doenças Endémicas Samuel Pessóa, Escola Nacional de Sauide Pública (Ensp/Fiocruz), Rua Leopoldo Bulhöes, 1480, Manguinhos, Rio de Janeiro, R, 21041, Brasil.

\section{Avaliação do Estado Nutricional} num Contexto de Mudança Sócio-Econômica: o Grupo Indígena Suruí do Estado de Rondônia, Brasil."

\author{
Carlos E.A. Coimbra Jr."* \\ Ricardo V. Santos"*
}

O presente trabalho discute os resultados de uma avaliação nutricional, realizada com 147 crianças de 0-8,9 anos da comunidade indigena Suruh, Parque Indigena Aripuanã, Rondônia. Os dados incluem antropometria, dosagem de niveis de hemoglobina e exame coproparasitologico. A reserva Surut localiza-se em uma região de intensa colonização e fluxo migratório. O grupo foi contactado pela Funai em 1969 $e$, nos últimos anos, envolveu-se ativamente no mercado regional, o que resultou em parcial abandono das atividades de subsistência tradicionais. Comparados com a população-referência do NCHS, os resultados indicam elevadas prevalencias de baixa altura para idade $(46,3 \%)$, peso para idade $(31,9 \%)$ e peso para altura (6,6\%). São também altas as prevalências de anemia $(71,2 \%)$ e parasitismo intestinal $(>75 \%)$. Os autores argumentam que a precariedade do estado nutricional das crianças Surui reflete carências alimentares, devido à redução da capacidade de produção de alimentos e inadequadas condições sanitárias presentes nas diversas aldeias.

\section{INTRODUÇÃO}

Apesar da reconhecida importância em termos de saúde pública, são poucos os trabalhos que incluem informações sobre o estado nutricional de populações indígenas brasileiras (cf. Dufour, 1991). Dentre esses, a maior parte foi 
realizada no Parque Indígena do Xingu (Baruzzi et al., 1977; Eveleth et al., 1974; Fagundes Neto et al., 1981; Franco, 1981; Morais, 1985), ou entre grupos que, pelo menos na época em que foram estudados, aderiam a padrões tradicionais de subsistência e de alimentação (Ayres \& Salzano, 1972; Black et al., 1977; Neel et al., 1964; Weinstein et al., 1967).

Historicamente, as sociedades indígenas brasileiras passam por acelerado processo de mudanças sócio-culturais e econômicas, uma vez em contato permanente com a sociedade nacional. Tais mudanças repercutem diretamente sobre os hábitos alimentares e o estado nutricional desses grupos, principalmente durante o processo inicial de inserção na economia de mercado regional (cf. Coimbra Jr., 1989; Flowers, 1983; Gross et al., 1979). Em decorrência disso, atividades básicas de subsistência tendem a ser parcial ou totalmente abandonadas, levando à redução na variabilidade alimentar e maior dependência em produtos industrializados. Portanto, populações indígenas constituem grupos de alta vulnerabilidade para a ocorrência de desnutrição protéico-energética, além de outras carências nutricionais, especialmente em crianças. Ao fenômeno de rápida transição, soma-se a elevada prevalência de doenças infecto-parasitárias, muitas delas de introdução recente, contribuindo para o agravamento do perfil de saúde dessas populações (Baruzzi et al., 1977; Chiappino, 1975; Coimbra Jr., 1987, 1989; Neel, 1974).

O objetivo deste trabalho é o de apresentar os resultados de uma avaliação nutricional, realizada entre os Suruí, grupo indígena localizado no Estado de Rondônia. Além de observações gerais sobre as estratégias de subsistência do grupo, o estudo incluiu levantamento antropométrico, dosagem de níveis de hemoglobina e exame parasitológico das fezes. Os resultados são interpretados à luz da história recente dos Suruí, caracterizada por acelerada transição sócio-cultural e econômica.

\section{MATERIAL E MÉTODOS}

\section{População Estudada}

O grupo indígena Suruí constituía uma população de cerca de 400 indivíduos em junho/julho de 1987, quando 
os dados aqui apresentados foram coletados. Os Suruí são falantes de uma língua classificada no tronco Tupí, família Mondé, e vivem distribuídos em dez aldeias, localizadas na Área Indígena Sete de Setembro, Parque Indígena Ari-

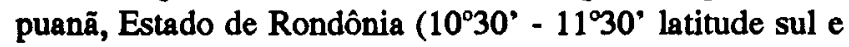
$60^{\circ} 30^{\prime}-61^{\circ} 30^{\prime}$ longitude oeste).

Os Suruí habitam uma região coberta por floresta de terra firme amazônica. Tradicionalmente, viviam em grandes malocas feitas com folhas da palmeira babaçu, onde residiam familias extensas. Atualmente, as mesmas vêm dando lugar a casas de madeira ou de pau-a-pique, onde predominam famílias nucleares (Coimbra Jr., 1985a, 1989). A economia tradicional do grupo é baseada na agricultura de corte-e-queima, suplementada pela caça, pesca e coleta de produtos silvestres. Dentre os principais cultivares presentes nas roças Suruí, destacam-se o milho, batata-doce, mandioca, cará e amendoim (Coimbra Jr., 1985d). Os animais de caça mais procurados são os porcos-do-mato, diversas espécies de macacos, tatus e aves de maior porte, como o mutum, cujubim e inhambus (Coimbra Jr., 1985b,d). Outras fontes de alimentos incluem variedades de mel de abelhas silvestres, palmitos, frutos e amêndoas, assim como alguns insetos, particularmente larvas de coleópteros e lepidópteros (Coimbra Jr., 1984, $1985 c$,d). Por serem os rios da região de pequeno a médio porte, a pesca assume papel secundário na economia do grupo (Coimbra Jr., 1985b).

Os primeiros relacionamentos pacíficos dos Suruí com a sociedade nacional datam de 1969 , quando o grupo foi contactado por sertanistas da Funai (Fundação Nacional do Indio). Epidemias de sarampo, gripe e tuberculose seguiram-se ao contacto, ocasionando a dizimação de mais da metade do contingente populacional original (Chiappino, 1975; Coimbra Jr., 1989). Em decorrência dessas epidemias, o grupo ficou de tal maneira desorganizado, tanto social como economicamente que, rapidamente, tornou-se muito dependente dos serviços da Funai, inclusive no tocante à provisão de alimentos e construção de casas.

Importantes mudanças voltaram a ocorrer na sociedade Suruí, a partir de 1983-1984. Com a retirada de colonos que invadiram o sul da reserva, duas das três aldeias então existentes fragmentaram-se em dez para ocupar e explorar 
os cafezais deixados pelos invasores. Os cafezais foram vistos pelos Suruí como um meio de inserção no mercado regional, possibilitando uma maior "independência" financeira. A concentração da força de trabalho nos cafezais levou a lavoura de subsistência a ocupar um plano inferior no cronograma de atividades do grupo. Também diminuiram de intensidade as atividades de pesca, caça e coleta. Tal situação resultou em quase total dependência em produtos industrializados na alimentação, os mesmos providos pela Funai ou adquiridos pelos Suruí com o dinheiro obtido da venda do café. Dentre os alimentos introduzidos, destacam-se o açúcar refinado, o arroz polido e as massas. De maneira geral, a dieta tornou-se predominantemente amilácea e monótona, não raro observando-se crianças alimentando-se unicamente de arroz com cará, mandioca ou outro tubérculo colhido nas roças. Tais mudanças repercutiram negativamente sobre o estado nutricional do grupo, manifestando-se no surgimento de casos de desnutrição aguda (Coimbra Jr., 1985d, 1989). A adoção da mamadeira e fórmulas infantis em substituição ao leite materno, possivelmente, também tiveram impacto negativo sobre o estado nutricional das crianças (Coimbra Jr., 1989).

Mais recentemente, devido aos baixos preços do café, os Suruí passaram a vender madeira extraída da reserva. Tal atividade não só levou ao afugentamento da caça que ainda restava nos arredores das aldeias, como também contribuiu para a destruição de várias espécies vegetais de importância na dieta tradicional do grupo (Coimbra Jr., $1985 \mathrm{c}, 1989)$.

O quadro geral de saúde da população é precário, como refletem os vários indicadores de morbi-mortalidade. Por exemplo, a taxa média de mortalidade infantil verificada entre os anos de 1979 e 1987 foi de aproximadamente 230 por mil. Tuberculose, malária, piodermites, gastrinterites e helmintoses são endêmicas entre os Suruí (Coimbra Jr., 1989; Coimbra Jr. \& Mello, 1981; Coimbra Jr. et al., 1985a,b; Santos et al., 1991).

\section{Metodologia Empregada}

Um total de 147 crianças Suruí de 0 a 8,9 anos de idade (83 meninos e 64 meninas) foram examinadas 
durante os meses de junho e julho de 1987, representando cerca de $95 \%$ do total de indivíduos na referida faixa etária. Já que a população Suruí é reduzida, não foi utilizada nenhuma técnica específica de amostragem, tendo-se procurado incluir no estudo o maior número de crianças. As idades foram obtidas a partir de cartões de vacinação ou registros existentes em postos da Funai. Infelizmente, isso nem sempre foi possível dada a falta de sistematização na coleta de dados demográficos por parte da Funai. Desse modo, as idades de parte das crianças tiveram que ser estimadas, utilizando-se, dentre outras técnicas, calendário envolvendo eventos locais persistentes na memória dos Suruí (Coimbra Jr., 1989).

As medidas antropométricas foram coletadas por um mesmo observador (Coimbra). O peso das crianças de idade até 5-6 anos foi medido utilizando-se uma balança portátil norte-americana fabricada por "Perspective Enterprises", com capacidade máxima de $25 \mathrm{~kg}$ e precisão de $100 \mathrm{~g}$. A balança era pendurada em uma viga e a criança, despida ou com o mínimo de roupa, colocada sentada em um suporte de nylon. Para as crianças mais velhas, utilizou-se uma balança alemã de plataforma marca "Seca", com capacidade máxima de $150 \mathrm{~kg}$ e precisão de $100 \mathrm{~g}$. Ambas as balanças eram periodicamente aferidas durante o trabalho de campo. Foi utilizado um antropômetro suíço marca "GPM", com precisão de $0,1 \mathrm{~cm}$. $O$ comprimento das crianças menores de dois anos foi medido em decúbito dorsal e a altura das mais velhas em posiçāo ortostática, com os pés juntos. e descalças.

De acordo com as recomendações da Organização Mundial da Saúde (WHO, 1986), os dados de peso e altura foram comparados com as curvas normalizadas da população-referência do NCHS ("National Center for Health Statistics") (Hamill et al., 1979). O cálculo dos desvios-padrão (valores " $Z$ ") para os indices antropométricos altura para idade (AI), peso para idade (PI) e peso para altura (PA) para cada criança foi realizado através de programa para microcomputadores distribuído pelo "Centers for Disease Control" (Jordan, 1986).

Seguindo Waterlow et al. (1977), considerou-se o ponto de corte de -2 desvios-padrão dos indices AI e PA para diferenciar crianças eutróficas das desnutridas (cf. também 
WHO, 1986). Dessa forma, crianças apresentando desvios-padrão de AI e PA inferiores a -2 são consideradas como tendo nanismo nutricional (desnutrição crônica ou "stunting") e atrofia nutricional (desnutrição aguda ou "wasting"), respectivamente.

$O$ perímetro braquial esquerdo (PB) foi medido utilizando-se uma fita metálica, com precisão de $0,1 \mathrm{~cm}$, no ponto mediano da distância entre o cotovelo e o processo acromial. A mesma fita foi usada na medição do perímetro cefálico (PC). A prega cutânea tricipital (PCT) foi medida na região posterior do braço esquerdo e na mesma altura do perímetro braquial, usando-se um compasso Lange (EUA) calibrado em milímetros. As recomendaçōes descritas em Weiner \& Lourie (1981) foram seguidas na medição do PB e da PCT. A partir dos dados de PB e PCT, foram estimados os índices "área muscular do braço" (AMB) e "área adiposa do braço" (AAB), de acordo com as fórmulas publicadas por Frisancho (1981). Os valores desses índices foram comparados com as curvas do NCHS (Frisancho, 1981; Frisancho \& Tracer, 1987).

Além do estudo antropométrico, foram dosados os níveis de hemoglobina em amostras de sangue obtidas, a partir de punção digital, através do método de Sahli (Lima et al., 1977). As dosagens de hemoglobina foram feitas por um mesmo observador (Coimbra) e considerou-se o valor de $11 \mathrm{~g} / 100 \mathrm{ml}$ como ponto de corte na definição de anemia. Realizou-se, também, um inquérito coproparasitológico qualitativo, através do método de sedimentação de Lutz. Os exames de fezes foram processados no laboratório do Núcleo de Medicina Tropical da Universidade de Brasília, a partir de amostras previamente fixadas em formol $10 \%$.

A análise estatística foi feita através do programa SPSS para microcomputadores (SPSS, 1989). A partir dos dados, foram obtidas estatísticas descritivas e os testes de qui-quadrado e Kolmogorov-Smirnov foram utilizados para detectar a existência de associações estatisticamente significantes entre variáveis específicas.

\section{RESULTADOS}

1. Antropometria Nutricional: Estatísticas descritivas 
dos índices antropométricos para os meninos e meninas Surui estão apresentadas nas Tabelas I e II, respectivamente.

\section{TABELA I}

Estatística Descritiva (Amostra, Média e Desvio-Padrão) da Antropometria dos Meninos Suruf [Altura (ALT), Peso (PESO), Perimetro Cefálico (PC) e Braquial (PB), Prega Cutânea Tricipital (PCT) e Área Muscular (AMB) e Adiposa do Braço (AAB)].

\begin{tabular}{crrrrrrr}
\hline \hline $\begin{array}{c}\text { IDADE } \\
\text { (anos) }\end{array}$ & $\begin{array}{r}\text { ALT } \\
(\mathrm{cm})\end{array}$ & $\begin{array}{c}\text { PESO } \\
(\mathrm{kg})\end{array}$ & $\begin{array}{c}\text { PC } \\
(\mathrm{cm})\end{array}$ & $\begin{array}{c}\text { PB } \\
(\mathrm{cm})\end{array}$ & $\begin{array}{c}\text { PCT } \\
(\mathrm{mm})\end{array}$ & $\begin{array}{r}\text { AMB } \\
\left(\mathrm{mm}^{2}\right)\end{array}$ & $\begin{array}{c}\text { AAB } \\
\left(\mathrm{mm}^{2}\right)\end{array}$ \\
\hline 0- 0,9 & & & & & & & \\
n & 13 & 13 & 12 & 13 & 13 & 13 & 13 \\
$\begin{array}{c}\text { Média } \\
\text { DP }\end{array}$ & 61,8 & 6,4 & 42,6 & 11,5 & 7,5 & 665,7 & 391,8 \\
$\begin{array}{c}\text { - 1,9 } \\
\text { n }\end{array}$ & 3,9 & 1,4 & 2,1 & 1,0 & 2,6 & 116,7 & 150,8 \\
Média & 68,8 & 3 & 3 & 3 & 3 & 3 & 3 \\
DP & 4,6 & 1,3 & 44,1 & 10,5 & 5,0 & 634,4 & 247,3 \\
$2-2,9$ & & & 1,4 & 2,0 & 132,5 & 112,9 \\
n & 7 & 7 & 7 & 7 & 7 & 7 & 7 \\
Média & 77,6 & 10,2 & 46,7 & 11,9 & 5,7 & 815,8 & 314,9 \\
DP & 6,2 & 1,4 & 2,1 & 0,7 & 1,7 & 108,9 & 97,8 \\
$3-3,9$ & & & & & & & \\
n & 5 & 5 & 5 & 5 & 5 & 5 & 5 \\
Média & 90,7 & 12,2 & 47,6 & 13,1 & 7,8 & 920,9 & 457,7 \\
DP & 4,4 & 1,6 & 1,8 & 0,9 & 2,7 & 234,3 & 132,0 \\
$4-4,9$ & & & & & & & \\
n & 9 & 9 & 9 & 9 & 9 & 9 & 9 \\
Média & 90,2 & 13,2 & 48,2 & 13,5 & 5,8 & 1087,7 & 367,4 \\
DP & 8,8 & 2,0 & 1,3 & 1,2 & 1,5 & 160,9 & 114,4 \\
$5-5,9$ & & & & & & & \\
n & 14 & 14 & 14 & 14 & 14 & 14 & 14 \\
Média & 98,6 & 15,8 & 49,9 & 14,0 & 6,2 & 1158,8 & 409,4 \\
DP & 10,3 & 3,3 & 1,6 & 1,2 & 1,8 & 158,8 & 137,5 \\
$6-6,9$ & & & & & & & \\
n & 12 & 12 & 11 & 11 & 12 & 11 & 11 \\
Média & 104,0 & 16,7 & 48,6 & 14,0 & 6,3 & 1151,7 & 404,9 \\
DP & 5,3 & 1,9 & 0,7 & 1,0 & 2,3 & 203,0 & 148,2 \\
$7-7,9$ & & & & & & & \\
n & 9 & 8 & 8 & 8 & 9 & 8 & 8 \\
Média & 115,1 & 19,8 & 49,1 & 15,1 & 6,3 & 1336,4 & 456,1 \\
DP & 7,8 & 1,9 & 1,9 & 0,5 & 1,5 & 161,4 & 94,9 \\
$8-8,9$ & & & & & & & \\
n & 11 & 6 & 9 & 10 & 11 & 10 & 10 \\
Média & 119,7 & 22,7 & 51,2 & 16,1 & 8,6 & 1424,5 & 653,2 \\
DP & 5,9 & 2,2 & 1,4 & 1,0 & 3,1 & 237,2 & 218,2 \\
\hline \hline & & & & & & & \\
\hline \hline
\end{tabular}




\section{TABELA II}

Estatística Descritiva (Amostra, Média e Desvio-Padrão) da Antropometria das Meninas Surú [Altura (ALT), Peso (PESO), Perímetro Cefálico (PC) e Braquial (PB), Prega Cutânea Tricipital (PCT) e Área Muscular (AMB) e Adiposa do Braço (AAB)].

\begin{tabular}{|c|c|c|c|c|c|c|c|}
\hline $\begin{array}{l}\text { DADE } \\
\text { (anos) }\end{array}$ & $\begin{array}{l}\text { ALT } \\
\text { (cm) }\end{array}$ & $\begin{array}{c}\text { PESO } \\
(\mathbf{k g})\end{array}$ & $\begin{array}{l}\mathrm{PC} \\
(\mathrm{cm})\end{array}$ & $\begin{array}{c}\mathrm{PB} \\
(\mathrm{cm})\end{array}$ & $\begin{array}{l}\text { PCT } \\
(\mathrm{mm})\end{array}$ & $\begin{array}{l}\text { AMB } \\
\left(\mathrm{mm}^{2}\right)\end{array}$ & $\begin{array}{c}\mathrm{AAB} \\
\left(\mathrm{mm}^{2}\right)\end{array}$ \\
\hline \multicolumn{8}{|l|}{$0-0,9$} \\
\hline $\mathbf{n}$ & 11 & 10 & 11 & 10 & 11 & 10 & 10 \\
\hline Média & 66,3 & 6,7 & 44,5 & 11,2 & 7,7 & 596,5 & 404,1 \\
\hline DP & 5,6 & 1,5 & 4,2 & 0,8 & 2,9 & 121,6 & 119,9 \\
\hline \multicolumn{8}{|l|}{$1-1,9$} \\
\hline $\mathrm{n}$ & 6 & 6 & 6 & 6 & 6 & 6 & 6 \\
\hline Média & 71,3 & 7,6 & 45,0 & 11,2 & 6,3 & 691,3 & 326,5 \\
\hline DP & 3,2 & 2,0 & 1,4 & 1,6 & 1,8 & 204,0 & 117,4 \\
\hline \multicolumn{8}{|l|}{$2-2,9$} \\
\hline $\mathrm{n}$ & 4 & 4 & 4 & 4 & 4 & 4 & 4 \\
\hline Média & 78,7 & 10,1 & 46,3 & 12,1 & 6,8 & 797,6 & 357,1 \\
\hline DP & 1,6 & 0,7 & 0,7 & 1,1 & 1,0 & 142,8 & 79,5 \\
\hline \multicolumn{8}{|l|}{$3-3,9$} \\
\hline $\mathrm{n}$ & 10 & 10 & 10 & 10 & 10 & 10 & 10 \\
\hline Média & 84,9 & 11,7 & 46,0 & 12,8 & 8,4 & 835,2 & 485,5 \\
\hline DP & 9,9 & 3,1 & 1,8 & 1,4 & 2,2 & 225,6 & 147,3 \\
\hline \multicolumn{8}{|l|}{$4-4,9$} \\
\hline $\mathrm{n}$ & 9 & 9 & 9 & 9 & 9 & 9 & 9 \\
\hline Média & 88,8 & 12,3 & 46,6 & 13,2 & 7,6 & 936,9 & 452,8 \\
\hline DP & 7,4 & 2,0 & 1,7 & 0,7 . & 1,8 & 130,3 & 110,9 \\
\hline \multicolumn{8}{|l|}{$5-5,9$} \\
\hline $\mathbf{n}$ & 7 & 7 & 7 & 7 & 7 & 7 & 7 \\
\hline Média & 96,2 & 14,6 & 48,7 & 14,0 & 5,9 & 1181,0 & 378,2 \\
\hline DP & 7,3 & 2,0 & 1,6 & 1,4 & 1,8 & 280,4 & 100,5 \\
\hline \multicolumn{8}{|l|}{$6-6,9$} \\
\hline $\mathbf{n}$ & 6 & 6 & 6 & 6 & 6 & 6 & 6 \\
\hline Média & 103,5 & 16,4 & 48,9 & 14,0 & 6,3 & 1149,7 & 404,9 \\
\hline DP & 6,3 & 1,9 & 1,1 & 0,9 & 1,0 & 164,2 & 148,2 \\
\hline \multicolumn{8}{|l|}{$7-7,9$} \\
\hline $\mathbf{n}$ & 8 & 7 & 7 & 7 & 8 & 7 & 7 \\
\hline Média & 110,4 & 17,0 & 50,2 & 14,8 & 6,6 & 1280,2 & 485,4 \\
\hline DP & 5,2 & 3,3 & 4,0 & 1,8 & 2,8 & 224,9 & 257,9 \\
\hline \multicolumn{8}{|l|}{$8-8,9$} \\
\hline n & 3 & 2 & 3 & 3 & 3 & 3 & 3 \\
\hline Média & 116,9 & 19,6 & 50,0 & 15,0 & 6,3 & 1340,6 & 442,5 \\
\hline DP & 8,7 & 2,8 & 1,1 & 0,3 & 0,6 & 63,7 & 42,1 \\
\hline
\end{tabular}


Como ilustrado nas Figuras I e II, as médias de altura e peso das crianças Suruí situam-se em torno do $3^{2}$ percentil da população-referência (NCHS). As Figuras I e II sugerem que as crianças passam a experimentar déficits de altura e peso, notadamente a partir do primeiro ano de vida. As figuras III e IV mostram que as crianças de ambos os sexos situam-se por volta do $50^{\circ}$ percentil para $o$ índice peso para altura. Conjuntamente, os resultados indicam que ocorrem déficits de altura para idade (AI) e de peso para idade (PI), ainda que as crianças Suruí aproximem-se da mediana da população-referência, no que tange a peso para altura (PA).

\section{FIGURA I}

Médias de Altura para Idade e Peso para Idade dos Meninos Surui Comparadas com o 3 Percentil da Referência (NCHS).

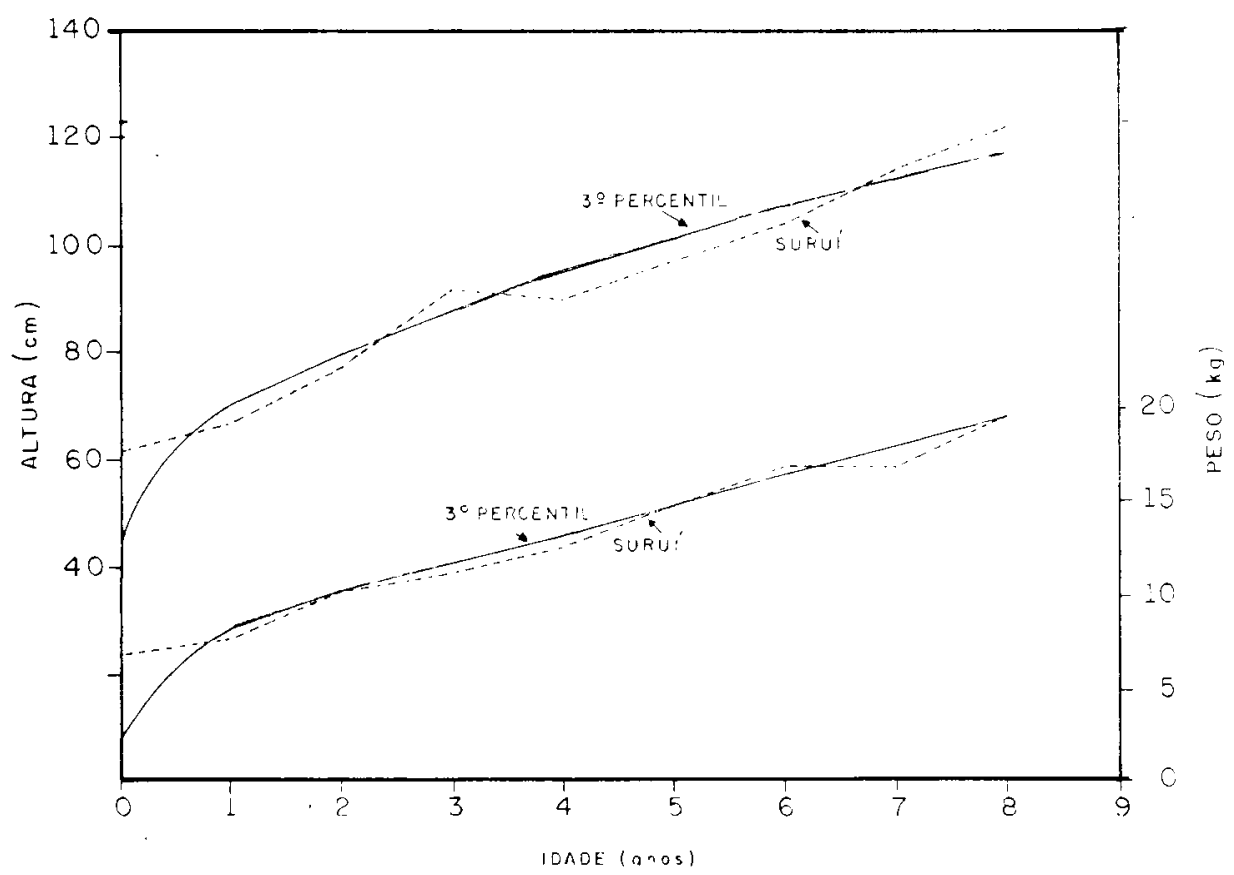


FIGURA II

Médias de Altura para Idade e Peso para Idade das Meninas

Suruí Comparadas com o $3^{\text {? }}$ Percentil da Referência (NCHS).

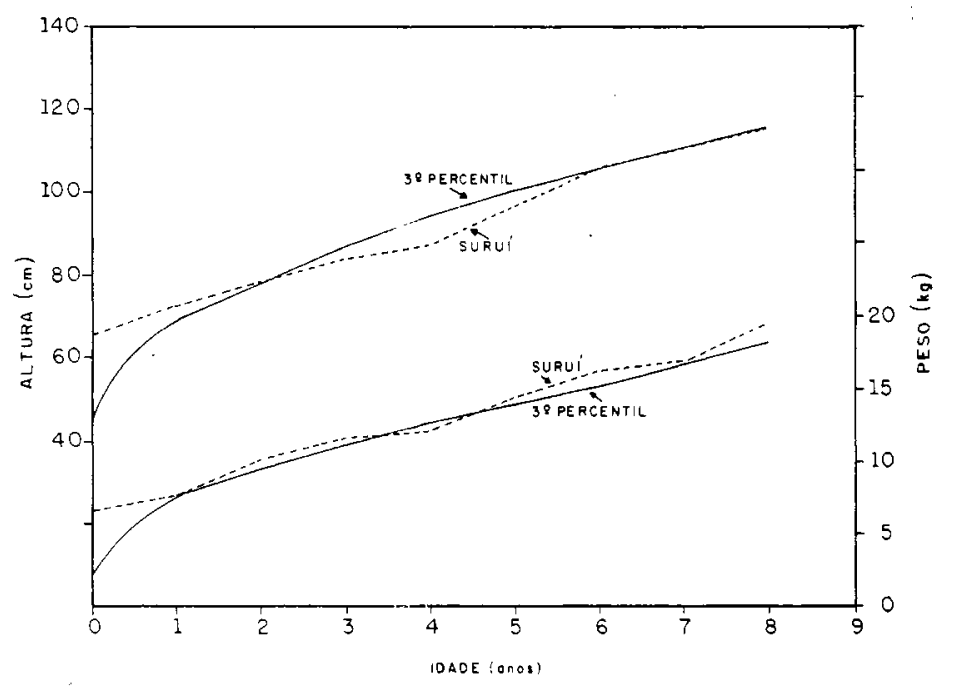

FIGURA III

Médias de Peso para Altura dos Meninos Surui Comparadas com o 50 Percentil da Referência (NCHS).

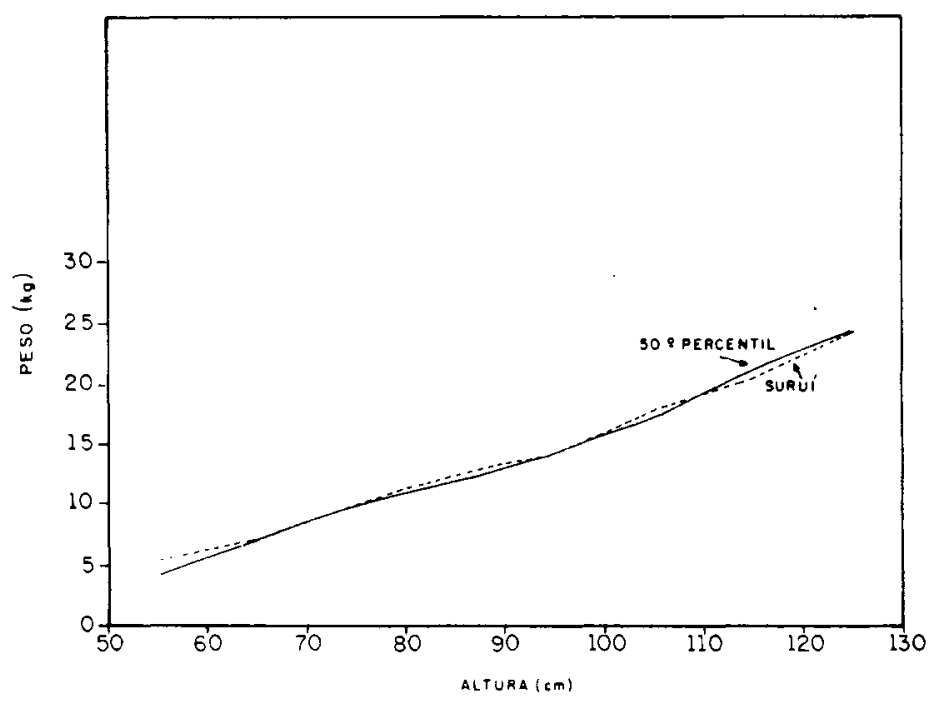

Cadernos de Saúde Pública, RJ, 7(4): 538-562, out/dez, 1991 


\section{FIGURA IV}

Médias de Peso para Altura das Meninas Surur Comparadas com o $50^{\circ}$ Percentil da Referência (NCHS).

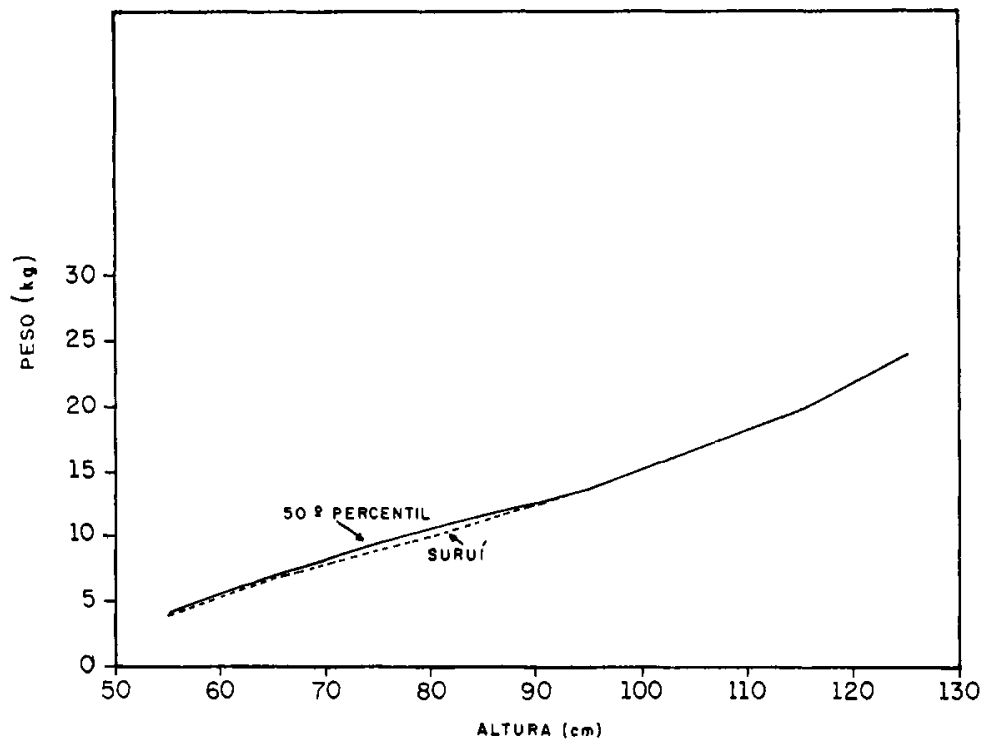

Estes resultados são sumarizados na Tabela III, onde as distribuições dos desvios-padrão (DP) (valores "Z") para os três indices antropométricos estão apresentados. Em relação à AI, 46,3\% das crianças situam-se abaixo de -2 DP. Quanto ao índice PA, 6,6\% da amostra encontra-se abaixo do ponto de corte de -2 DP. A Tabela III também indica que $31,9 \%$ das crianças apresentam valores abaixo de -2 DP para PI. Testes de Kolmogorov-Smirnov foram conduzidos para verificar se as distribuições das freqüências absolutas de meninos e meninas nos diversos intervalos de desvios-padrão para AI, PI e PA são semelhantes. Já que as células de " $1 \leq \mathrm{DP}<2$ " e "DP $\geq 2$ " (Tabela III) são bastante reduzidas no caso de AI e PI, tais faixas foram combinadas para efeito do teste; quanto à PA, as faixas "DP $\leq-2$ " e "-2 $<$ DP $\leq-1$ " foram combinadas, assim como " $1 \leq \mathrm{DP}<2$ " e "DP $\geq 2$ ". Os resultados dos três testes de Kolmogorof-Smirnov indicam que, ao nível de 5\%, os meninos e meninas Suruí não diferem na distribuição de freqüências absolutas, segundo intervalos de desvios-padrão para AI, PI ou PA. 


\section{TABELA III}

Frequência Absoluta e Relativa, Segundo Intervalos

dos Desvios-Padrão (DP) (Valores " $Z$ ")

dos Indices Antropométricos Altura para Idade, Peso para Idade

e Peso para Altura das Crianças Suruí de 0 a 8,9 Anos

em Relação à População-Referência (NCHS),

Segundo Sexo $(M=$ Masculino, $F=$ Feminino e $T=$ Total $)$.

\begin{tabular}{|c|c|c|c|c|c|c|c|c|}
\hline \multicolumn{9}{|c|}{ Indices Antropométricos } \\
\hline \multicolumn{3}{|c|}{$\begin{array}{c}\text { Altura } p / \text { idade } \\
\text { (AI) }\end{array}$} & \multicolumn{3}{|c|}{$\begin{array}{c}\text { Peso } \mathrm{p} / \text { idade } \\
\text { (PI) }\end{array}$} & \multicolumn{3}{|c|}{$\begin{array}{c}\text { Peso p/ altura } \\
\text { (PA) }\end{array}$} \\
\hline $\mathbf{M}$ & $\mathbf{F}$ & $\mathbf{T}$ & $\mathbf{M}$ & $\mathrm{F}$ & $\mathrm{T}$ & $\mathbf{M}$ & $\mathbf{F}$ & $\mathbf{T}$ \\
\hline \multicolumn{9}{|l|}{$D P \leq-2$} \\
\hline 42 & 26 & 68 & 25 & 19 & 44 & 3 & 6 & 9 \\
\hline $50,6 \%$ & $40,6 \%$ & $46,3 \%$ & $32,5 \%$ & $31,1 \%$ & $31,9 \%$ & $4,0 \%$ & $9,8 \%$ & $6,6 \%$ \\
\hline \multicolumn{9}{|l|}{$-2<D P \leq-1$} \\
\hline 18 & 23 & 41 & 28 & 25 & 53 & 6 & 6 & 12 \\
\hline $21,7 \%$ & $35,9 \%$ & $27,9 \%$ & $36,4 \%$ & $41,0 \%$ & $38,4 \%$ & $8,0 \%$ & $9,8 \%$ & $8,8 \%$ \\
\hline \multicolumn{9}{|l|}{$-1<D P \leq 0$} \\
\hline 13 & 6 & 19 & 20 & 11 & 31 & 35 & 26 & 61 \\
\hline $15,7 \%$ & $9,4 \%$ & $12,9 \%$ & $26,0 \%$ & $18,0 \%$ & $22,5 \%$ & $46,7 \%$ & $42,6 \%$ & $44,9 \%$ \\
\hline \multicolumn{9}{|l|}{$0<\mathrm{DP}<1$} \\
\hline 5 & 4 & 9 . & 2 & 5 & 7 & 24 & 18 & 42 \\
\hline $6,0 \%$ & $6,3 \%$ & $6,1 \%$ & $2,6 \%$ & $8,2 \%$ & $5,1 \%$ & $32,0 \%$ & $29,5 \%$ & $30,9 \%$ \\
\hline \multicolumn{9}{|l|}{$1 \leq \mathrm{DP}<2$} \\
\hline 4 & 3 & 7 & 2 & - & 2 & 7 & 5 & 12 \\
\hline $4,8 \%$ & $4,7 \%$ & $4,8 \%$ & $2,6 \%$ & & $1,4 \%$ & $9,3 \%$ & $8,2 \%$ & 8,89 \\
\hline \multicolumn{9}{|l|}{$\mathrm{DP} \geq 2$} \\
\hline 1 & 2 & 3 & - & 1 & 1 & - & - & - \\
\hline $1,2 \%$ & $3,1 \%$ & $2,0 \%$ & & $1,6 \%$ & $0,7 \%$ & & & \\
\hline \multicolumn{9}{|l|}{ Total } \\
\hline 83 & 64 & 147 & 77 & 61 & 138 & 75 & 61 & 136 \\
\hline $100 \%$ & $100 \%$ & $100 \%$ & $100 \%$ & $100 \%$ & $100 \%$ & $100 \%$ & $100 \%$ & $100 \%$ \\
\hline
\end{tabular}


As médias do perimetro cefálico das crianças Suruí encontram-se abaixo dos valores publicadas para crianças inglesas (Ousnted et al., 1975) (Tabelas I e II).

Os dados de composição corporal estão sumarizados nas Tabelas I e II. Comparados com a população do NCHS, as médias do perímetro braquial, prega cutânea tricipital e áreas muscular e adiposa para idade dos meninos e meninas Suruí situam-se em torno ou abaixo do $5^{2}$ percentil da referência. Os valores de área muscular para altura de ambos os sexos localizam-se, também, por volta do $5^{2}$ percentil da população-referência.

As freqüências relativas de nanismo e atrofia nutricional segundo idade são apresentadas na Tabela IV. Analisar-se-ão os resultados com sexos combinados, já que as amostras por faixa etária são reduzidas (cf. Tabelas I e II). Em termos de nanismo nutricional, as crianças Suruí de $0,0-0,9$ anos já apresentam prevalências da ordem de $20,8 \%$, indicando que o processo de redução de crescimento começa bastante cedo. Há um primeiro pico na prevalência de nanismo nas crianças $1,0-1,9$ anos $(55,6 \%)$, tendendo a aumentar nas idades seguintes até atingir um segundo pico na faixa etária 4,0-4,9 anos $(77,8 \%)$. A partir dessa idade, as prevalências tendem a decrescer. Com relação à atrofia nutricional, observa-se uma prevalência bastante acentuada nas crianças de $1,0-1,9$ anos $(44,4 \%)$ e valores medianos nas faixas $0,0-0,9 ; 3,0-3,9$ e $4,0-4,9$ anos.

2. Hemoglobina: os resultados indicam que $71,2 \%$ (99) da amostra de 139 crianças apresentam níveis de hemoglobina abaixo de $11 \mathrm{~g} / 100 \mathrm{ml}$ de sangue, caracterizando um quadro de alta prevalência de anemia (Tabela IV). Considerando os sexos separadamente, 51 meninos $(65,4 \%)$ e 48 meninas $(78,7 \%)$ apresentam-se anêmicas. Testes de qui-quadrado $\left(\chi^{2}=2,34 ; 1\right.$ g.l.; $\left.p=0,13\right)$ indicam que não há associação estatisticamente significante ao nivel de $5 \%$ entre sexo e prevalência de anemia.

Quanto às prevalências de anemia segundo idade, as mesmas tendem a ser elevadas ( $>50 \%) \mathrm{em}$ todas as faixas de idade, sendo particularmente altas nas crianças menores de cinco anos (>70\%), chegando a $100 \%$ nas de $3,0-3,9$ anos (Tabela IV). 
TABELA IV

Amostras e Frequêencias Relativas de Nanismo Nutricional, Atrofia Nutricional e Anemia, Segundo Faixa Etária.

\begin{tabular}{cccc}
\hline \hline $\begin{array}{c}\text { Faixas etárias } \\
\text { (anos) }\end{array}$ & $\begin{array}{c}\text { Nanismo } \\
\text { nutricional }\end{array}$ & $\begin{array}{c}\text { Atrofia } \\
\text { nutricional }\end{array}$ & Anemia \\
\hline $0,0-0,9$ & $\mathrm{n}=24$ & $\mathrm{n}=21$ & $\mathrm{n}=24$ \\
& $20,8 \%$ & $9,5 \%$ & $79,2 \%$ \\
$1,0-1,9$ & $\mathrm{n}=9$ & $\mathrm{n}=9$ & $\mathrm{n}=9$ \\
& $55,6 \%$ & $44,4 \%$ & $77,8 \%$ \\
$2,0-2,9$ & $\mathrm{n}=11$ & $\mathrm{n}=11$ & $\mathrm{n}=11$ \\
& $45,5 \%$ & - & $72,7 \%$ \\
$3,0-3,9$ & $\mathrm{n}=15$ & $\mathrm{n}=15$ & $\mathrm{n}=15$ \\
& $53,3 \%$ & $6,7 \%$ & $100 \%$ \\
$4,0-4,9$ & $\mathrm{n}=18$ & $\mathrm{n}=18$ & $\mathrm{n}=18$ \\
& $77,8 \%$ & $5,6 \%$ & $72,2 \%$ \\
$5,0-5,9$ & $\mathrm{n}=21$ & $\mathrm{n}=21$ & $\mathrm{n}=21$ \\
& $57,1 \%$ & - & $57,1 \%$ \\
$6,0-6,9$ & $\mathrm{n}=18$ & $\mathrm{n}=18$ & $\mathrm{n}=18$ \\
& $50,0 \%$ & - & $61,1 \%$ \\
$7,0-7,9$ & $\mathrm{n}=17$ & $\mathrm{n}=15$ & $\mathrm{n}=14$ \\
& $35,3 \%$ & - & $64,3 \%$ \\
$8,0-8,9$ & $\mathrm{n}=14$ & $\mathrm{n}=8$ & $\mathrm{n}=9$ \\
& $28,6 \%$ & - & $55,6 \%$ \\
Total & $\mathrm{n}=147$ & $\mathrm{n}=136$ & $\mathrm{n}=139$ \\
& $46,3 \%$ & $6,6 \%$ & $71,2 \%$ \\
\hline \hline
\end{tabular}

3. Parasitologia das Fezes: a Tabela $\mathrm{V}$ resume os resultados dos 124 exames coproparasitológicos realizados. Dentre os helmintos, destacam-se os ancilostomideos e Ascaris lumbricoides, por apresentarem as maiores prevalências, respectivamente de $76,6 \%$ e $64,5 \%$. As prevalências de Trichuris trichiura, Enterobius vermicularis, Hymenolepis nana e Strongyloides stercoralis variam de $15,3 \%$ a $41,1 \%$. Quanto aos protozoários parasitas, Giardia lamblia e Entamoeba histolytica apresentam prevalências de $46,8 \%$ e $21,8 \%$, respectivamente. As seguintes espécies de protozoários comensais foram detectados: Entamoeba coli, lodamoeba bütschlii, Chilomastix mesnili e Endolimax nana. 
Testes de qui-quadrado (total de 12 testes) indicaram não haver associação estatisticamente significante ao nível de 5\% entre os resultados do exames (positivo e negativo) e sexo para cada uma das espécies apresentadas na Tabela V.

\section{TABELA V}

Frequuências Relativas de Crianças Surul 0-8.9 Anos Positivas nos Exames Coproparasitológicos, de Acordo com Sexo.

\begin{tabular}{lccc}
\hline \multirow{2}{*}{ Parasitas e comensais } & Meninos & Meninas & Total \\
\cline { 2 - 4 } & $(\mathrm{n}=66)$ & $(\mathrm{n}=58)$ & $(\mathrm{n}=124)$ \\
\hline Ancilostomidae & $68,0 \%$ & $77,6 \%$ & $76,6 \%$ \\
Ascaris lumbricoides & $63,6 \%$ & $65,5 \%$ & $64,5 \%$ \\
Strongyloides stercoralis & $34,8 \%$ & $48,3 \%$ & $41,1 \%$ \\
Trichuris trichiura & $30,3 \%$ & $24,1 \%$ & $27,4 \%$ \\
Enterobius vermicularis & $15,2 \%$ & $15,5 \%$ & $15,3 \%$ \\
Hymenolepis nana & $22,7 \%$ & $15,5 \%$ & $19,4 \%$ \\
Giardia lamblia & $45,5 \%$ & $48,3 \%$ & $46,8 \%$ \\
Entamoeba histolytica & $25,8 \%$ & $17,2 \%$ & $21,8 \%$ \\
Entamoeba coli & $65,2 \%$ & $70,7 \%$ & $67,7 \%$ \\
Iodamoeba bütschlii & $62,1 \%$ & $63,8 \%$ & $62,9 \%$ \\
Chilomastix mesnili & $18,2 \%$ & $15,5 \%$ & $16,9 \%$ \\
Endolimax nana & $18,2 \%$ & $24,1 \%$ & $31,0 \%$ \\
\hline \hline
\end{tabular}

\section{DISCUSSÃO}

Uma das premissas da avaliação antropométrica do estado nutricional, utilizando uma população-referência como o NCHS, é de que tal conjunto de curvas possa ser satisfatoriamente aplicado a qualquer população, independentemente de sua constituição genética (Habicht et al., 1974; Waterlow et al., 1977; WHO, 1986). Segundo tal perspectiva, crianças bem nutridas de diferentes grupos populacionais tendem a crescer de maneira similar, 
sugerindo, portanto, que fatores genéticos exercem menor influência sobre o crescimento do que os de ordem ambiental. Ainda que atraente por permitir a utilização de uma única referência, parece haver exceções que podem limitar a generalização dessa idéia (Davies, 1988; Eveleth \& Tanner, 1990; Garn \& Clark, 1976). Davies (1988), por exemplo, argumenta que crianças de origem asiática possam ser geneticamente menores em termos de estatura; Garn \& Clark (1976), por sua vez, sugerem a utilização de curvas de crescimento específicas na avaliação nutricional de crianças negras norte-americanas.

A análise da antropometria nutricional dos Suruí indica que $46,3 \%$ e $31,9 \%$ das crianças situam-se abaixo de -2 desvios-padrão para os índices altura para idade (AI) e peso para idade (PI), respectivamente. Por outro lado, a maioria está dentro da faixa de normalidade para o índice peso para altura (PA), ainda que tenha sido detectada uma prevalência de $6,6 \%$ de baixo peso para altura. Além disso, os resultados também indicam que as médias do perímetro cefálico das crianças Suruí encontram-se abaixo daqueles da referência utilizada.

A elevada prevalência de nanismo nutricional entre os Suruí (46,3\%) está de acordo com o esperado em face das precárias condições sanitárias e alimentares presentes na área indígena. Este resultado reflete o acúmulo de episódios adversos em termos de saúde e que impediram o crescimento esquelético nos niveis esperados, produzindo uma população de crianças de baixa estatura. Para efeito de comparação, as prevalências de nanismo nutricional reportadas em estudos realizados com populaçōes não-indígenas e de baixa renda de diversas regiões do Brasil são geralmente inferiores a 20\% (e.g., Anjos, 1989; Batista-Filho et al., 1981; Gross et al., 1987; Reichenheim \& Harphan, 1990), podendo elevarem-se a níveis próximos ou superiores a $40 \%$ naquelas regiões mais pobres e carentes, como o Nordeste e Norte do país (Batista-Filho, 1986; Giugliano et al., 1981; WHO, 1987). A comparação dos resultados Suruí com de outras populações reforça a idéia aventada por antropólogos de que o processo de assimilação de populações indígenas faz-se com sua inserção nos níveis mais baixos do sistema de estratificação sócio-econômica da sociedade brasileira 
(cf. Coimbra Jr., 1989; Oliveira, 1978), refletindo em níveis mais elevados de desnutrição e mortalidade infantil do que as mais altas médias nacionais.

Obviamente que as condiçōes adversas de crescimento físico das crianças Suruí dificultam qualquer discussão acerca de potencialidades ligadas a fatores de ordem genética (Davies, 1988; Eveleth \& Tanner, 1990). De qualquer modo, a prevalência de baixa altura para idade da ordem te $46,3 \%$ é um resultado esperado em face das precarias condições de saúde da população.

A alta prevalência de atrofia nutricional $(6,6 \%)$ entre os Suruí também está de acordo com as condiçōes sócio-econômicas e sanitárias presentes na área indígena. No período estudado, a situação alimentar nas aldeias estava de tal forma grave que chegou a mobilizar a população indígena, assim como a Funai, no sentido de organizar um programa emergencial de suplementação alimentar e implantação de roças. Infelizmente o programa não atingiu seus objetivos. A ocorrência de desnutrição aguda encontra suporte nos baixos valores dos índices antropométricos de composição corporal (perímetro braquial, prega cutânea tricipital e áreas muscular e adiposa do braço), os quais indicam reduzidas reservas protéicas e energéticas. A prevalência de atrofia nutricional entre as crianças Surui é superior aos valores detectados em estudos com crianças indígenas (Black et al., 1977; Fagundes-Neto et al., 1981) e não-indígenas de baixa renda (Anjos, 1989; Gross et al., 1987; Reichenheim \& Harpham, 1990).

Além da elevada prevalência de desnutrição protéico-energética, os resultados deste inquérito revelam frequiências igualmente altas de anemia e parasitoses intestinais. O quadro de anemia é particularmente grave, pois atinge cerca de $71 \%$ das crianças estudadas. A dieta pobre e monótona adotada pelo grupo, aliada à elevada freqüência de parasitas intestinais, em particular os ancilostomídeos, explicam, pelo menos parcialmente, os níveis de anemia encontrados (Variyam \& Banwell, 1982). Afora sua associação direta com o quadro de anemia, os parasitas intestinais relacionam-se com o processo de desnutrição de forma mais global, à medida que provocam perda de apetite, má absorção de nutrientes ou mesmo a perda direta desses provocada por diarréias e/ou vômitos 
(Farthing et al., 1986; Tripathy et al., 1971; Variyam \& Banwell, 1982).

Não foram encontradas associaçōes estatisticamente significantes entre nenhuma das variáveis consideradas (índices antropométricos, anemia e parasitismo intestinal) e sexo das crianças. De certa forma, tais achados são contrários às informações disponiveis para os Suruí. Com base em dados etnográficos e de estrutura demográfica, Coimbra Jr. (1989) sugere que ocorre entre os Suruí um processo semelhante ao que Scrimshaw (1984) denomina de "subinvestimento" nas crianças do sexo feminino. Tal processo incluiria tratamento diferenciado em termos de assistência médica, alimentar, física e/ou emocional. Neste contexto, esperar-se-ia que o cuidado diferencial destinado aos meninos se refletisse nos indicadores nutricionais.

A consideração das prevalências de desnutrição segundo idade revela a existência de um padrão condizente com o já descrito para outras populaçōes, qual seja, um aumento do estresse nutricional no período que corresponde com o desmame (cf. Gordon et al., 1963). Coimbra Jr. (1989) menciona que o periodo de amamentação das crianças Suruí é prolongado, não sendo incomum observar-se crianças de dois a três anos sugando o seio materno. Portanto, o aumènto significativo nos níveis de desnutrição aguda, como evidenciado pelo indicador peso para altura, no segundo ano de vida da criança Suruí, deve estar relacionado com o período no qual o leite materno torna-se menos proeminente na dieta do lactente. Além disso, é de se esperar que episódios de diarréia devam aumentar no período de desmame, pelo contato maior da criança com objetos não apropriadamente lavados e consumo de água e alimentos contaminados. A longo prazo, é também no segundo ano de vida que o acúmulo em déficits de crescimento linear faz-se mais aparente, acúmulo este que se estende pela vida da criança Suruí, como pode ser verificado pelos níveis de desnutrição crônica revelada pelo indicador altura para idade.

Este estudo evidencia inadequações estruturais da sociedade Suruí frente ao acelerado processo de mudanças sócio-culturais e econômicas que ora se verifica, agravando a já precária situação de saúde e nutricional que caracteriza a população. As inter-relações entre mudanças sócio- 
-culturais e econômicas : com a deterioração do estado nutricional das crianças Surui são certamente complexas. Dentre alguns fatores observados durante o periodo de mudanças e que, certamente, estão relacionados ao quadro nutricional aqui reportado, destacam-se a redução da capacidade de produção de alimentos tradicionais, a monotonia da nova dieta e a adoção de técnicas inadequadas no preparo de alimentos industrializados (Coimbra Jr., 1989). Além destes fatores, as elevadas prevalências de gastroenterites, infeç̧ões respiratórias agudas, parasitoses intestinais, malária e outros processos infecciosos (cf. Coimbra Jr., 1989; Coimbra Jr. \& Mello, 1981; Coimbra Jr. et al., 1985a,b; Santos et al., 1991) repercutem sobre o estado nutricional das crianças à medida em que provocam falta de apetite, diarréia, má absorção de nutrientes, vômitos e fadiga, dentre outros sintomas (Martorell, 1980; Mata et al., 1977; Nabarro et al., 1988).

Ainda que o estudo da biologia humana de populações indigenas brasileiras tenha avançado enormemente em décadas recentes (Salzano \& Callegari-Jacques, 1988), faz-se necessárias pesquisas mais abrangentes de forma a elucidar as inter-relações entre mudanças sócio-culturais e econômicas e o estado de saúde desses grupos. Os resultados do inquérito nutricional dos Suruí demonstram elevadas prevalências de desnutrição protéico-energética, anemia e parasitismo intestinal. Pesquisas ao longo da mesma linha com outras comunidades indígenas brasileiras evidenciariam se o quadro detectado para os Suruí encontra paralelos em outras comunidades também experimentando rápida transiçăo nas diversas esferas de suas vidas. Tal linha de pesquisa constitui-se em importante fonte de dados necessária no monitoramento de mudanças em comunidades indígenas.

This paper details the results of a nutritional assessment conducted with 147 Surui Amerindian children 0-8.9 years of age from the Aripuanã Indian Park, Rondônia, Brazil. The data include anthropometry, hemoglobin 
concentration levels, and stool examinations. The Surui reservation is located in an area which experienced intensive government-oriented colonization and immigration over the past three decades. The group was contacted by the Brazilian Indian Foundation (FUNAI) in 1969 and, recently, became more involved in the regional market economy, which led tc oartial abandonment of traditional subsistence strategies. Compared to international reference curves (NCHS), the results indicate high levels of low height for age (46.3\%), weight for age (31.9\%) and weight for height $(6.6 \%)$. Anemia (71.2\%) and intestinal parasitism (over $75 \%)$ are also common. The authors argue that the precarious nutritional status of the Surui children reflects nutritional problems due to a reduction in the Surui food production system as well as inadequate sanitary conditions of the various villages.

\section{AGRADECIMENTOS}

Os autores agradecem a comunidade Suruí pela colaboração e interesse manifestado pelo trabalho e a Fundação Nacional do Indio (Funai) pelo apoio durante a pesquisa de campo. Agradecemos, também, ao Dr. Luiz A. dos Anjos, Escola Nacional de Saúde Pública, Fiocruz, pela leitura crítica dos originais. Quaisquer erros são, obviamente, de nossa total responsabilidade.

\section{REFERENCIAS BIBLIOGRÁFICAS}

ANJOS, L. A. - Indices antropométricos e estado nutricional de escolares de baixa renda de um municipio do Estado do Rio de Janeiro (Brasil): um estudo piloto. Revista de Saúde Pública, 23: 221-229, 1989.

AYRES, M. \& SALZANO, F. M. - Health status of Brazilian Cayapó Indians. Tropical and Geographical Medicine, 24: 178-185, 1972.

BARUZZI, R. G.; MARCOPITO, L. F.; SERRA, M. L. C.; SOUZA, F. A. A. \& STABILE, C. - The Kren-Akorore: A recently contacted indigenous tribe. In: Health and Disease in Tribal Societies (K. Elliot \& J. Whelan, orgs.), pp. 179-211, Amsterdam, Elsevier, 1977. 
BATISTA-FILHO, M. - Nutrição, Alimentação e Agricultura no Nordeste Rrasileiro - Subsídios para uma Politica de Extensāo Rural. Brasilia, FAO/ Embrater, 1986.

BATISTA-FILHO, M.; LUCENA, M. A. F. \& COELHO, H.A. L. Protein-calorie malnutrition in three Brazilian State capitals: São Luís, Recife, and São Paulo. Bulletin of the Pan American Health Organization, 15: 231-240, 1981.

BLACK, F. L.; HIERHOLZER, W. J.; BLACK, D. P.; LAMM, S. H. \& LUCAS, L. - Nutritional status of Brazilian Kayapó Indians. Human Biology, 49: 139-153, 1977.

CHIAPPINO, J. - The Brazilian Indigenous Problem and Policy: the Aripuanã Park. Série Documentos № 19. Copenhaguem, International Work Group for Indigenous Affairs/Genebra, Information Center for Indigenous Affairs in the Amazon Region, 1975.

COIMBRA JR., C. E. A. - Estudos de ecologia humana entre os Suruí do Parque Indígena Aripuanã, Rondônia. 1. O uso de larvas de coleópteros (Bruchidae e Curculionidae) na alimentação. Revista Brasileira de Zoologia, 2: 35-47, 1984.

A habitação Suruí e suas implicações epidemiológicas. In: Adaptação à Enfermidade e sua distribuição entre Grupos Indígenas da Bacia Amazônica (Ibánez-Novion, M. A. \& Ott, A. M. T., orgs.), pp. 120-138, Brasília, Centro de Estudos e Pesquisas em Antropologia Médica/Belém, Museu Paraense Emilio Goeldi, 1985a.

Estudos de ecologia humana entre os Suruí de Rondônia. Elementos de etnozoologia. Boletim do Museu Paraense Emilio Goeldi (Antropologia), 2: 9-36, $1985 \mathrm{~b}$.

Estudos de ecologia humana entre os Suruí de Rondônia. Plantas de importância econômica. Boletim do Museu Paraense Emilio Goeldi (Antropologia), 2: 37-55, 1985 c.

Estudos de ecologia humana entre os Suruí de Rondônia. Aspectos alimentares., Boletim do Museu Paraense Emilio Goeldi (Antropologia), 2: 57-87, 1985d.

O sarampo entre sociedades indígenas brasileiras e algumas considerações sobre a prática da saúde pública entre estas populações. Cadernos de Saúde Pública, 3: 22-37, 1987.

From Shifting Cultivation to Coffee Farming: The Impact of Change on the Health and Ecology of the Surui in the Brazilian Amazon. Ph.D. Dissertation, Bloomington, Department of Anthropology, Indiana University (E.U.A.), 1989. 
COIMBRA JR., C. E. A. \& MELLO, D. A. - Enteroparasitoses e Capillaria sp. entre o grupo indígena Suruí, Parque Indígena Aripuanã, Rondônia. Memórias do Instituto Oswaldo Cruz, 76: 299-302, 1981.

COIMBRA JR., C. E. A.; SANTOS, R. V. \& TANUS, R. Estudos epidemiológicos entre grupos indígenas de Rondônia. I. Piodermites e portadores inaparentes de Staphylococcus sp. na boca e nariz entre os Suruí e Karitiana. Revista do Instituto de Medicina Tropical de Säo Paulo, 27: 13-19, 1985a.

COIMBRA JR., C. E. A.; SANTOS, R. V.; TANUS, R. \& INHAM, T. M. - Estudos epidemiológicos entre grupos indígenas de Rondônia. II. Bactérias enteropatogênicas $e$ gastrenterites entre as Suruí e Karitiana. Revista da Fundação Sesp, 30: 111-119, 1985b.

DAVIES, D. P. - The importance of genetic influences on growth in early childhood with particular reference to children of Asiatic origin. In: Linear Growth Retardation in Less Developed Countries (Waterlow, J.C., org.), Nestlé Nutrition Workshop Series, vol. 14, pp. 75-90, New York, Vevey/ Raven Press, 1988.

DUFOUR, D. L. - Diet and nutritional status of Amerindians: a review of the literature. Cadernos de Saúde Pública, 7: 481$502,1991$.

EVELETH, P. B.; SAlZANO, F. M. \& LIMA, P. E. - Child growth and adult physique in Brazilian Xingu Indians. American Journal of Physical Anthropology, 41: 95-102, 1974.

EVELETH, P. B. \& TANNER, J. V. - Worldwide Variation in Human Growth, 2: Ed., Cambridge, Cambridge University Press, 1990.

FAGUNDES-NETO, U.; BARUZZI, R. G.; WEHBA, J.; MORAIS, M. B. \& CAINELLI, M., SILVESTRINI, W. S.; Observations of the Alto Xingu Indians (Central Brazil) with special reference to nutritional evaluation in children. American Journal of Clinical Nutrition, 34: 2229-2235, 1981.

FARTHING, M. J. G.; URRUTIA, J. J. \& KRONMAL, R. A.; MATA, L. - Natural history of Giardia infections of infants and children in rural Guatemala and its impact on physical growth. American Journal of Clinical Nutrition, 43: 395$-405,1986$.

FLOWERS, N. M. - Seasonal factors in subsistence, nutrition, and child growth in a Central Brazilian Indian community. In: Adaptive Responses of Native Amazonians (R. B. Hames \& W. H. Vickers, orgs.), pp. 357-390, New York, Academic Press, 1983. 
FRANCO, L. J. - Aspectos Metabólicos da População Indigena do Alto Xingu (Brasil Central). Tese de Doutorado, São Paulo, Departamento de Medicina Preventiva, Escola Paulista de Medicina, 1981.

FRISANCHO, A. R. - New norms of upper limb fat and muscle areas for assessment of nutritional status. American Journal of Clinical Nutrition, 34: 2540-2545, 1981.

FRISANCHO, A. R. \& TRACEK, D. P. - Standards of arm muscle by stature for the assessment of nutritional status of children. American Journal of Physical Anthropology, 73: 459-465, 1987.

GARN, S. M. \& CLARK, D. C. - Problems in the nutritional assessment of Black individuals. American Journal of Public Health, 66: 262-267.

GIUGLIANO, R.; GIUGLIANO, L. G. \& SHRMMPTON, R. Estudos nutricionais das populações rurais da Amazônia. I. Várzea do Rio Solimões. Acta Amazonica, 11: 773-788, 1981.

GORDON, J. E.; CHITKARA, I. D. \& WYON, J. B. - Weanling diarthea. American Journal of Medical Sciencies, 245: 345$-377,1963$.

GROSS, D. R.; EITEN, G.; FLOWERS, N. M.; LEOI, F. M.; RITTER, M. L. \& WERNER, D. W. - Ecology and acculturation among native peoples of Central Brazil. Science, 206: 1043-1050, 1979.

GROSS, R.; STANGE, M.; SOLOMONS, N.W.; ESQUTVEL, I.R. \& OLTERRSDORF, U. - The influence of economic deterioration in Brazil on the nutritional status of children in Rio de Janeiro, Brazil. Ecology of Food and Nutrition, 19: 265-279, 1987.

HABICHT, J. P.; MARTORELL, R.; YARBROUGH, C.; KLEIN, R.E. \& MALINA, R. M. - Height and weight standards for preschool children: how relevant are ethnic differences in growth potential? Lancet, 1: 611-615, 1974.

HAMILL, P. V. V.; DRZD, T. A.; JOHNSON, C. L.; REED, R. B; ROCHE, A. F. \& MOORE, W. M. - Physical growth: National Center for Health Statistics percentiles. American Journal of Clinical Nutrition, 32: 607-629, 1979.

JORDAN, M. D. - Anthropometric Software Package. Tutorial Guide and Handbook, Version 3.0. Atlanta, Centers for Disease Control, 1986.

LIMA, A. O; SOARES, J. B.; GRECO, J. B.; GALIZZI, J. \& CANÇADO, J. R. - Métodos de Laboratório Aplicados à Clínica, 5: Ed., Rio de Janeiro, Guanabara-Koogan, 1977. 
MARTORELL, R. - Interrelationships between diet, infectious disease and nutritional status. In: Social and Biological Predictors of Nutritional Status, Physical Growth, and Behavioral Development (L. S. Greene \& F. Johnston, orgs.), pp. 81-106, New York, Academic Press, 1986.

MATA, L. J.; KRONAL, R. A.; URRUTIA, J. J. \& GARCIA, B. - Effects of infection on food intake and the nutritional state: perspectives as viewed from the village. American Journal of Clinical Nutrition, 30: 1215-1227, 1977.

MORAIS, M. B. - Estado Nutricional de Crianças Indias do Alto Xingu e Avaliação do Perímetro Braquial no Diagnóstico da Desnutrição Protéico-Calórica. Tese de Doutorado, São Paulo, Departamento de Medicina Preventiva, Escola Paulista de Medicina, 1985.

NABARRO, D.; HOWARD, P.; CASSELS, C.; PANT, M.; WIJGA, A. \& PADFIELD, N. - The importance of infectious and environmental factors as possible determinants of growth retardation in children. In: Linear Growth Retardation in Less Developed Countries (L. C. Waterlow, org.), Nestlé Nutrition Workshop Series, vol. 14, pp. 165-183, New York, Vevey/Raven Press, 1988.

NEEL, J. V. - Control of disease among Amerindians in cultural transition. Bulletin of the Pan American Health Organization, 8: 205-211, 1974.

NEEL, J. V.; SALZANO, F. M.; JUNQUEIRA, P. C.; KEITER, F. \& MAYBURY-LEWIS, D. - Studies on the Xavante Indians of the Brazilian Mato Grosso. American Journal of Human Genetics, 16: 52-140, 1964.

OLIVEIRA, R. C. - Sociologia do Brasil Indigena. 2" Ed., Brasilia, Editora da Universidade de Brasília, 1978.

OUNSTED, M.; MOAR, V. A. \& SCOTT, A. - Head circumference charts updated. Archives of Disease in Childhood, 60: 936-939, 1985.

REICHNHEIM, M. E. \& HARPHAM, T. - Perfil intracomunitário da deficiência nutricional: estudo de crianças abaixo de 5 anos numa comunidade de baixa renda do Rio de Janeiro (Brasil). Revista de Saúde Pública, 24: 69-79, 1990.

SALZANO, F. M. \& CALLEGARI-JACQUES, S. - South American Indians: A Case Study in Evolution. Oxford, Clarendon Press, 1988.

SANTOS, R. V.; LINHARES, A. C. \& COIMBRA JR., C. E. A. - Estudos epidemiológicos entre grupos indigenas de Rondônia. IV. Inquérito sorológico para rotavírus entre os Suruí e Karitiána. Revista de Saúde Pública, 25: 103-106, 1991. 
SCRIMSHAW, S. C. M. - Infanticide in human populations: societal and individual concerns. In: Infanticide, Comparative and Evolutionary Perspectives (G. Hausfater \& S. B. Hrdy, orgs.), pp. 439-462, New York, Aldine, 1984.

SPSS (Statistical Package for the Social Sciences) - SPSS/PC + 2.0 Base Manual. Chicago, SPSS Inc., 1989.

TRIPATHY, K.; GONZÁLES, F.; LOTERO, H. \& BOLANOS, O. - Effects of ascariasis on human nutrition. American Journal of Tropical Medicine and Hygiene, 20: 212-218, 1971.

VARIYAM, E. P. \& BANWELL, J. G. - Hookworm disease: nutritional implications. Reviews of Infectious Diseases, 4: 830-835, 1982.

WATERLOW, C.; BUZINA, R.; KELLER, W.; LANE, J. M.; NICHAMAN, M. Z. \& TANNER, J. M. - The presentation and use of height and weight data for comparing the nutritional status of groups of children under the age of 10 years. Bulletin of the World Health Organization, 55: 489-498, 1977.

WEINER, J. S. \& LOURIE, J. A. (orgs.) - Practical Human Biology. London, Academic Press, 1981.

WEINSTEIN, E. D.; NEEL, J. V. \& SALZANO, F. M. - Further studies on the Xavante Indians. VI. The physical status of the Xavantes of Simões Lopes. American Journal of Human Genetics, 19: 532-542, 1967.

WHO (World Health Organization) - Use and interpretation of anthropometric indicators of nutritional stat us. Bulletin of the World Health Organization, 64: 929-941, 1986.

Nutrition. Global surveillance through anthropometric measurements. Weekly Epidemiological Record, 9: 57-59, 1987. 